\title{
Two-edged sword: how activation of the "proto-oncogene" yes-associated protein 1 in lung squamous cell carcinoma can surprisingly inhibit tumor growth
}

\author{
Thomas Andl ${ }^{1}$, Claudia D. Andl ${ }^{1}$, Yuhang Zhang ${ }^{2}$ \\ ${ }^{1}$ Burnett School of Biological Sciences, University of Central Florida, Orlando, FL, USA; ${ }^{2}$ Division of Pharmaceutical Sciences, College of Pharmacy, \\ University of Cincinnati, Cincinnati, OH, USA \\ Correspondence to: Dr. Thomas Andl. Burnett School of Biomedical Sciences, University of Central Florida, 12722 Research Parkway, Orlando, FL \\ 32826, USA. Email: thomas.andl@ucf.edu. \\ Provenance: This is an invited Editorial commissioned by the Section Editor Chunlin Ou (Cancer Research Institute of Central South University, \\ Changsha, China). \\ Comment on: Huang H, Zhang W, Pan Y, et al. YAP Suppresses Lung Squamous Cell Carcinoma Progression via Deregulation of the DNp63-GPX2 \\ Axis and ROS Accumulation. Cancer Res 2017;77:5769-81.
}

Submitted Aug 01, 2018. Accepted for publication Oct 01, 2018.

doi: $10.21037 /$ jtd.2018.10.11

View this article at: http://dx.doi.org/10.21037/jtd.2018.10.11

The Hippo signaling pathway is a highly conserved pathway that regulates cell proliferation and apoptosis. The core components of the pathway, a cascade of protein serine kinases, are commonly present in most eukaryotic organisms (1). In animals, the pathway is associated with limiting growth and thereby controlling e.g., organ size (2). The major target of Hippo signaling is the transcriptional regulator yes-associated protein 1 (YAP1). Inhibition of YAP1 via phosphorylation by kinases of the Hippo signaling pathway prevents the translocation of YAP1 from the cytoplasm to the nucleus and subsequent activation of YAP1 target genes (2). A plethora of evidence shows that inhibition of Hippo signaling and activation of YAP1 is oncogenic. Furthermore, overexpression and nuclear localization of YAP1 has been detected in multiple human cancers, supporting the idea that the YAP1 is an oncogene (3). In this general framework, the Hippo signaling pathway is considered to be a tumor suppressor pathway that inhibits the activation of the proto-oncogene YAP1.

In lung squamous cell carcinomas (SCCs) this dogma that Hippo signaling is a tumor suppressor pathway and oncogenic functions of YAP1 are activated is supported by the identification of mutations in core genes that can affect Hippo signaling and decreased expression of Hippo kinases. The mutations occur at relatively low rates and indicate inhibition of Hippo signaling and activation of
YAP1 (4) [e.g., YAP1 at 1\%, large tumor suppressor kinase $1 / 2(L A T S 1 / 2)$ at $3.5 \%$, WW domain-containing protein 1 $(W W C 1)$ at $3.2 \%$, FAT atypical cadherin 4 (FAT4) at 7\%, catenin at $14 \%]$. Furthermore, the majority of human nonsmall cell lung cancers (NSCLCs), including lung SCCs, have drastically reduced levels of LATS2 expression (5) a kinase of the Hippo pathway that directly inactivates YAP1 by phosphorylating the S217 site in YAP1.

However, a few reports have contradicted the straightforward storyline of YAP1 as an oncogene $(6,7)$. Dr. Hongbin Ji's group recently performed a study (Huang et al.) suggesting that YAP1 may be a more complex player in cancer than previously anticipated (8). In their report, Dr. Ji's laboratory provided evidence that YAP1 may function in an unorthodox way in a subset of lung squamous cell cancers, in which its activation may lead to the inhibition of cancer growth (8).

Interestingly, Huang et al.'s primary interest had not been in Hippo signaling per se but in the discovery of new drugs that can target lung SCCs. Their drug screen initially discovered digitoxin and digoxin to be new candidates for anti-cancer drugs because of their significant inhibitory effects on the viability of human lung SCC cell line L78 (8). Further in vivo testing showed that digitoxin is able to inhibit tumor growth in lung SCC cell line xenografts and patient-derived xenograft (PDX) models. Since digitoxin 
has been suggested to interact with YAP1 (9) and had been used to turn on YAP1 activity (10), Huang et al. speculated that a major pathway altered by digitoxin in lung SCCs may be Hippo/YAP1 signaling. Indeed, treatment of lung SCC cell lines in vitro and in vivo with digitoxin significantly reduced the phosphorylation of YAP1 and increased its nuclear translocation and activation.

Interestingly and surprisingly, overexpression of a mutant YAP1, YAP1 (S217A), that cannot be phosphorylated at the critical site S217, led to similar outcomes as digitoxin treatment itself and suggests that YAP1 does not always function as an oncogene and is important for the cytotoxic effect of digitoxin in lung SCCs. Even more strikingly, in Huang et al.'s experiments, suppression of LATS1/2, the kinases that phosphorylate S217 in YAP1, or the inhibition of the Hippo upstream kinases STK3/4, also resulted in growth suppression, despite their general recognition as tumor suppressor genes. Previous studies in lung adenocarcinoma cell lines had similar findings: siRNAmediated suppression of LATS2 inhibits proliferation (11). What is puzzling here is that in general many NSCLCs, including lung SCCs, already have very low expression levels of LATS1/2 (5). So why does further reduction of their expression cause growth inhibition? In addition, we and others have observed the dependency of YAP1 levels on the presence of LATS1/2 or STK3/4 $(12,13)$. However, based on the experiments of Huang et al. it is unclear whether inhibition of LATS1/2 and STK3/4 are equivalent to constitutive activation of YAP1 signaling using YAP1(S217A).

Huang et al. then asked the obvious question: how is it possible that Hippo signaling is upside down in lung SCCs? Since domains within YAP1 that mediate its transcriptional activity were required for its growth suppressive function, the authors interrogated the transcriptome of YAP1 (S217A)-overexpressing cells. Surprisingly, genes associated with removal of reactive oxygen species (ROS), e.g., GPX2, were reduced upon YAP1 (S217A) overexpression. The authors concluded that digitoxin treatment or inhibition of Hippo signaling and activation of YAP1 resulted in excessive ROS production, which in turn led to growth inhibition and death (Figure 1). Worth to mention, YAP1 can be induced by increased levels of ROS during neurogenesis, e.g., knockout of the uncoupling protein 2 (UCP2) in mitochondria results in increased ROS levels, which in turn induce nuclear accumulation of YAP1 and increased YAP1dependent proliferation (14).

And this relationship between YAP1 and ROS is an emerging theme in Hippo/YAP1 signaling. In cardiomyocytes a YAP1/FOXO1 complex activated catalase genes and SOD2 to reduce ROS production (15). Furthermore, YAP1 and the Drosophila homologue Yorkie can dramatically reduce ROS production in several cancer cell lines and in in vivo animal models through transcriptional regulation of a set of mitochondrial genes and SOD2 (16). There is now also mounting evidence that ROS, e.g., derived from mitochondrial activity, can affect Hippo signaling and YAP1 activity in different cell types (16). In Drosophila, a major result of the transcriptional activation may be mediated through regulators of mitochondrial fusion opa 1 and mitochondrial assembly Marf (16). Here YAP1/Yorkie have clearly protective functions: ROS activate YAP1 and YAP1 induces the transcription of ROS-eliminating enzymes, which in turn reduce ROS levels (Figure 1) $(15,17)$. However, the consequences of YAP1 activation can still differ dramatically and either induce proliferation (15) or apoptosis (17) probably depending how well ROS levels are contained.

In the case of Huang et al., the induction of YAP 1 by ROS remained unexplored despite indications that some cardiac glycosides may be able to induce ROS. The authors themselves showed dramatic increases of ROS in response to digitoxin but did not explore whether ROS by themselves are able to induce YAP1 activation in lung cancer cells. It remains to be seen if ROS activation is indeed a typical function of digitoxin or if the opposite is the norm (18). To add another layer of complexity, WWTR1, a YAP1 related protein, can also be regulated by ROS. At least in HEK293T cells, WWTR1 is clearly up-regulated within 1 hour of $\mathrm{H}_{2} \mathrm{O}_{2}$ exposure, while YAP1 is reduced (19). Interestingly, WWTR1 contains several cysteines that are responsive to oxidation under oxidative stress conditions while YAP1 does not contain any cysteines at all, suggesting that WWTR1 could function as a redox sensor (19). In any case, the relationship between Hippo/YAP1/WWTR1 signaling and ROS is an exciting field which offers new insights into the crucial role Hippo signaling occupies in cell biology.

To test whether manipulation of YAP1 activity through digitoxin could be of any use in treating human lung SCCs, Huang et al. tested two lung SCC PDXs models, one with high levels of YAP1 expression and the other with a low YAP1 level. As expected based on their previous results, the PDX model with low YAP1 expression showed upregulation of nuclear YAP1, growth inhibition and elevated ROS activity when treated with digitoxin, while the other 


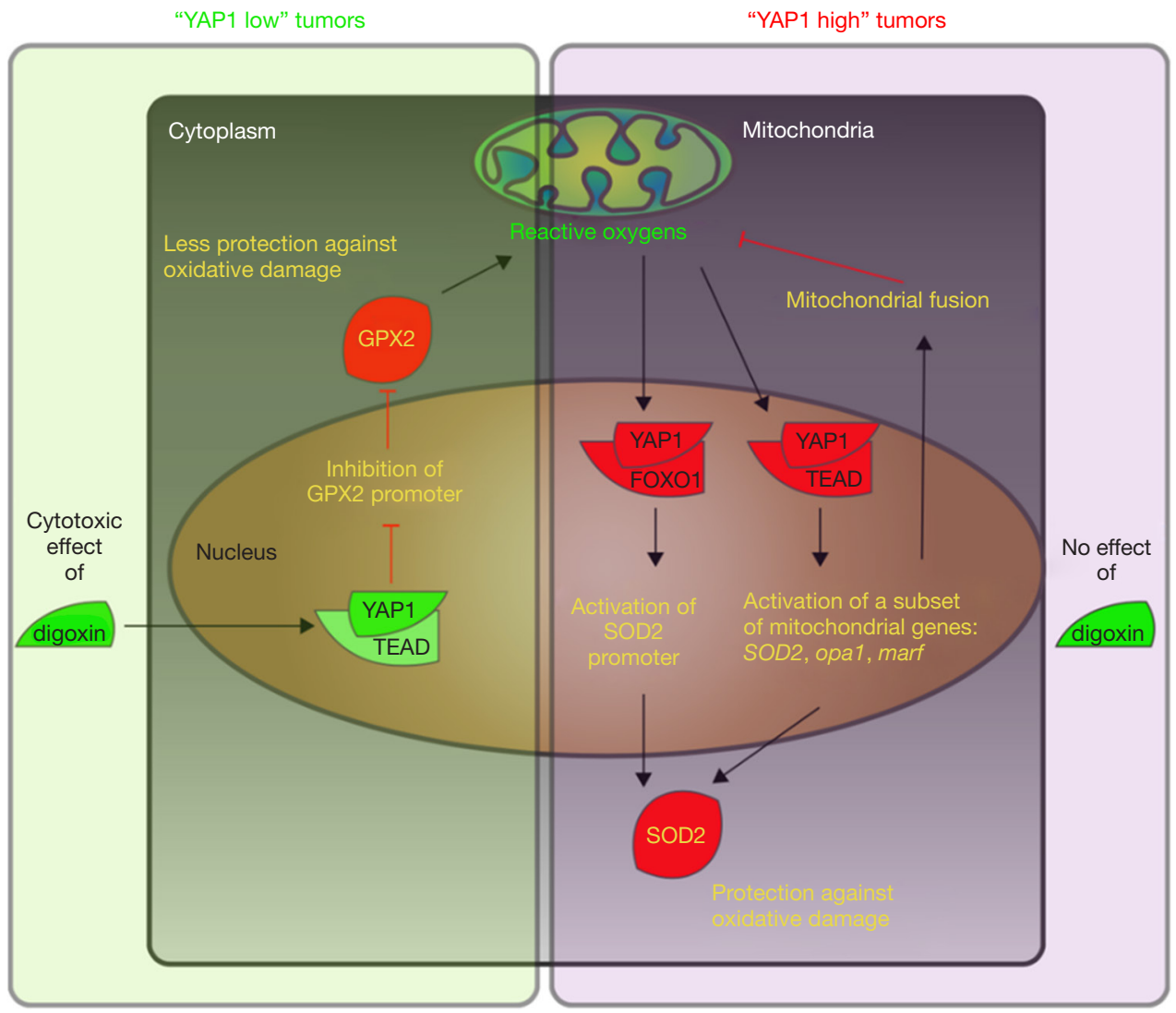

Figure 1 Model of differential response to digoxin in "YAP1 low" and "YAP1 high" tumors. "YAP1 high" tumors show nuclear expression of YAP1 and are adapted to the activity of YAP1. "YAP1 low" tumors express little YAP1 but respond to digoxin with an increase in nuclear YAP1 which eventually results in a toxic increase of ROS production due to inhibition of GPX2 and other enzymes that normally protect the cell against elevated ROS. On the other hand, "YAP1 high" tumors are well adapted to elevated ROS levels. In striking contrast to "YAP1 low" tumors in which YAP1 activation leads to more ROS production, YAP1 activation leads to the activation of genes such as SOD2 that control ROS levels. Furthermore, ROS themselves may actually induce YAP1 activity in these "YAP1 high" tumors. Therefore, in "YAP1 high" tumors YAP1 exhibits its classical oncogenic properties while in YAP1 low tumors, YAP1 can function as a tumor suppressor once activated, e.g., by digoxin treatment. YAP1, yes-associated protein 1; ROS, reactive oxygen species.

PDX model did not respond well. The data suggest that two different molecular classes of lung SCCs may exist: one that has activated YAP1 through mutation or alternative mechanisms within the Hippo pathway and the other that can still activate YAP1 "normally" but the cells are not adapted to YAP1 activation and are unprepared for elevated ROS production. Data on nuclear YAP1 expression support this grouping with about $25-60 \%$ of the lung SCCs expressing nuclear YAP1 (20-22). Interestingly, the rough framework of the Ji laboratory's work on YAP1 in lung cancer proposes that YAP1 activation is associated with TP63 down-regulation and an adenocarcinoma phenotype (22). With that in mind, the question arises, how do the $25-60 \%$ of lung SCCs (20-22) with activated nuclear YAP1 maintain their SCC phenotype? How have they adapted?

Another critical question is: will digitoxin treatment of YAP1-low tumors be successful? Probably not. Most likely the tumor cells will eventually adapt to the YAP1 activation and learn to deal with the consequences of elevated ROS, i.e., develop resistance against the drug (e.g., switch to an adenocarcinoma differentiation type or mutate the Hippo/ YAP1 pathway). Furthermore, since digitoxin and digoxin are relatively toxic in humans and have been associated with elevated cancer rates (23), their application as lung SCC drugs or drugs against YAP1-low cancers may be 
disappointing.

In this regard the perplexing question remains unaddressed on whether YAP1 activation is the main culprit of digitoxin toxicity in lung SCC cells. Digitoxin exhibits anti-cancer activity in many cell lines, including cell lines where YAP1 activity is probably beneficial. Are there really two independent mechanisms of digitoxin toxicity, one relying on an atypical YAP1 function and one on a more "classical" Na-K-ATPase inhibition (24)? It is unclear from Huang et al.'s findings whether the "classical" digitoxin cytotoxicity mechanisms are actually active in lung SCC cells. If YAP1 is indeed the main gateway through which digitoxin exerts its anti-lung SCC effects, this would be remarkable.

The dual role of YAP1 in lung cancer proposed by the Ji laboratory has previously been discussed in colon cancer (25). Ou et al. have explored how YAP1 can have opposite effects in cancer cells and suggested that YAP1 can mediate the effects of other signaling pathways beyond Hippo signaling. YAP1's "flexibility" to bind several other transcription factors and cytoplasmic proteins may play a crucial role in whether YAP1 is oncogenic or tumor suppressive (25). This is plausible when keeping in mind the staggering number of 271 confirmed YAP1 interactors based on BioGRID (https://thebiogrid.org/115684/ summary/homo-sapiens/yap1.html). How these complex interactions of YAP 1 are at work in lung cancer is still unclear. Interestingly, YAP1 does what Ou et al. have suggested: YAP1 can interfere with pathways well outside Hippo signaling, e.g., p63 signaling, and lead to fundamental and surprising changes in cell behavior (8). Since YAP1 has this versatile interactome, targeting YAP1 for the purpose of cancer therapy may result in unintended consequences. If YAP1 can function as an oncogene as well as a tumor suppressor, finding the right balance, tools and approaches to fine-tune YAP activity may be difficult to achieve without deep knowledge of a patient's molecular tumor classification.

The work of the Ji laboratory highlights the complexities and classification potential that YAP1 itself brings to NSCLC. To have, or not to have YAP1, that is the question that may have tremendous implications for the therapy of lung cancer patients in the near future. Defining the context-dependent interactors of YAP1, whether they are TEADs, TP73, SMADs, or other factors may greatly enhance the ability to define the role of YAP 1 in the different types of NSCLC and their tumor-associated cell populations.

\section{Acknowledgements}

None.

\section{Footnote}

Conflicts of Interest: The authors have no conflicts of interest to declare.

\section{References}

1. Hilman D, Gat U. The Evolutionary History of YAP and the Hippo/YAP Pathway. Mol Biol Evol 2011;28:2403-17.

2. Yu FX, Zhao B, Guan KL. Hippo Pathway in Organ Size Control, Tissue Homeostasis, and Cancer. Cell 2015;163:811-28.

3. Sun Z, Xu R, Li X, et al. Prognostic Value of YesAssociated Protein 1 (YAP1) in Various Cancers: A MetaAnalysis. PLoS One 2015;10:e0135119.

4. Li C, Gao Z, Li F, et al. Whole Exome Sequencing Identifies Frequent Somatic Mutations in Cell-Cell Adhesion Genes in Chinese Patients with Lung Squamous Cell Carcinoma. Sci Rep 2015;5:14237.

5. Malik SA, Khan MS, Dar M, et al. Molecular Alterations and Expression Dynamics of LATS1 and LATS2 Genes in Non-Small-Cell Lung Carcinoma. Pathol Oncol Res 2018;24:207-14.

6. Yuan M, Tomlinson V, Lara R, et al. Yes-associated protein (YAP) functions as a tumor suppressor in breast. Cell Death Differ 2008;15:1752-9.

7. Wang H, Du YC, Zhou XJ, et al. The dual functions of YAP-1 to promote and inhibit cell growth in human malignancy. Cancer Metastasis Rev 2014;33:173-81.

8. Huang H, Zhang W, Pan Y, et al. YAP Suppresses Lung Squamous Cell Carcinoma Progression via Deregulation of the DNp63-GPX2 Axis and ROS Accumulation. Cancer Res 2017;77:5769-81.

9. Inada A, Nakanishi T, Konoshima T, et al. Anti-tumor promoting activities of natural products. II. Inhibitory effects of digitoxin on two-stage carcinogenesis of mouse skin tumors and mouse pulmonary tumors. Biol Pharm Bull 1993;16:930-1.

10. Casey FP, Pihan E, Shields DC. Discovery of Small Molecule Inhibitors of Protein-Protein Interactions Using Combined Ligand and Target Score Normalization. J Chem Inf Model 2009;49:2708-17.

11. Luo SY, Sit KY, Sihoe AD, et al. Aberrant large tumor suppressor 2 (LATS2) gene expression correlates with 
EGFR mutation and survival in lung adenocarcinomas. Lung Cancer 2014;85:282-92.

12. Luan L, Shi J, Yu Z, Andl T. The major miR-31 target genes STK40 and LATS2 and their implications in the regulation of keratinocyte growth and hair differentiation. Exp Dermatol 2017;26:497-504.

13. Chung C, Kim T, Kim M, et al. HIPPO-Foxa2 signaling pathway plays a role in peripheral lung maturation and surfactant homeostasis. Proc Natl Acad Sci U S A 2013;110:7732-7.

14. Ji F, Shen T, Zou W, et al. UCP2 Regulates Embryonic Neurogenesis via ROS-Mediated Yap Alternation in the Developing Neocortex. Stem Cells 2017;35:1479-92.

15. Shao D, Zhai P, Del Re DP, et al. A functional interaction between Hippo-YAP signalling and FoxO1 mediates the oxidative stress response. Nat Commun 2014;5:3315.

16. Nagaraj R, Gururaja-Rao S, Jones KT, et al. Control of mitochondrial structure and function by the Yorkie/YAP oncogenic pathway. Genes Dev 2012;26:2027-37.

17. Lehtinen MK, Yuan Z, Boag PR, et al. A conserved MST-FOXO signaling pathway mediates oxidative-stress responses and extends life span. Cell 2006;125:987-1001.

18. Dixit D, Ghildiyal R, Anto NP, et al. Chaetocin-induced ROS-mediated apoptosis involves ATM-YAP1 axis and JNK-dependent inhibition of glucose metabolism. Cell Death Dis 2014;5:e1212.

19. Silva LN, Pessoa MT, Alves SL, et al. Differences of lipid membrane modulation and oxidative stress by digoxin and 21-benzylidene digoxin. Exp Cell Res 2017;359:291-8.

20. Gandhirajan RK, Jain M, Walla B, et al. Cysteine S-Glutathionylation Promotes Stability and Activation of the Hippo Downstream Effector Transcriptional Coactivator with PDZ-binding Motif (TAZ). J Biol Chem 2016;291:11596-607.

21. Wang Y, Dong Q, Zhang Q, et al. Overexpression of yes-associated protein contributes to progression and poor prognosis of non-small cell lung cancer. Cancer Sci 2010;101:1279-85.

22. Gao Y, Zhang W, Han X, et al. YAP inhibits squamous transdifferentiation of Lkb1-deficient lung adenocarcinoma through ZEB2-dependent DNp63 repression. Nat Commun 2014;5:4629.

23. Osman MH, Farrag E, Selim M, et al. Cardiac glycosides use and the risk and mortality of cancer; systematic review and meta-analysis of observational studies. PLoS One 2017;12:e0178611.

24. Prassas I, Karagiannis GS, Batruch I, et al. Digitoxininduced cytotoxicity in cancer cells is mediated through distinct kinase and interferon signaling networks. Mol Cancer Ther 2011;10:2083-93.

25. Ou C, Sun Z, Li S, et al. Dual roles of yes-associated protein (YAP) in colorectal cancer. Oncotarget 2017;8:75727-41.

Cite this article as: Andl T, Andl CD, Zhang Y. Two-edged sword: how activation of the "proto-oncogene" yes-associated protein 1 in lung squamous cell carcinoma can surprisingly inhibit tumor growth. J Thorac Dis 2018;10(Suppl 33):S3870S3874. doi: 10.21037/jtd.2018.10.11 